\title{
Democratization in a passive dendritic tree: an analytical investigation
}

\author{
Y Timofeeva*, $\quad$ S J Cox ${ }^{\dagger}, \quad$ S Coombes ${ }^{\ddagger}$ and $\mathrm{K}$ Josić $^{\S}$
}

\begin{abstract}
One way to achieve amplification of distal synaptic inputs on a dendritic tree is to scale the amplitude and/or duration of the synaptic conductance with its distance from the soma. This is an example of what is often referred to as "dendritic democracy". Although well studied experimentally, to date this phenomenon has not been thoroughly explored from a mathematical perspective. In this paper we adopt a passive model of a dendritic tree with distributed excitatory synaptic conductances and analyze a number of key measures of democracy. In particular, via moment methods we derive laws for the transport, from synapse to soma, of strength, characteristic time, and dispersion. These laws lead immediately to synaptic scalings that overcome attenuation with distance. We follow this with a Neumann approximation of Green's representation that readily produces the synaptic scaling that democratizes the peak somatic voltage response. Results are obtained for both idealized geometries and for the more realistic geometry of a rat CA1 pyramidal cell. For each measure of democratization we produce and contrast the synaptic scaling associated with treating the synapse as either a conductance change or a current injection. We find that our respective scalings agree up
\end{abstract}

${ }^{*}$ Department of Computer Science, University of Warwick, Coventry, CV4 7AL, UK. email: y.timofeeva@warwick.ac.uk

${ }^{\dagger}$ Department of Computational and Applied Mathematics, Rice University, Houston, TX 77005 , USA. email: cox@rice.edu

${ }_{\ddagger}^{\ddagger}$ School of Mathematical Sciences, University of Nottingham, Nottingham, NG7 2RD, UK. email: stephen. coombes@nottingham.ac.uk

${ }^{\S}$ Department of Mathematics, University of Houston, Houston, TX 77204-3008, USA. email: josic@math.uh.edu 
to a critical distance from the soma and we reveal how this critical distance decreases with decreasing branch radius.

Keywords: dendritic democracy, cable equation, shunts

\section{Introduction}

There is experimental evidence that synaptic efficacy increases as a function of distance from the soma [14, 3, 15, 20, 4, 21, 23], at least in CA1 hippocampal cells and spinal motoneurons. This is most likely due to increases in receptor density and/or vesicle release probability. One way to achieve such an increase in synaptic efficacy is through local anti spike timing-dependent plasticity $[26,27]$. Distance dependent synaptic scaling is just one of several mechanisms for achieving so-called "dendritic democracy" whereby the spatially extended single neuron can compensate for dendritic attenuation [18]. Other mechanisms for boosting somatic response to distal inputs include sub-threshold resonance (via active currents such as $\mathrm{I}_{h}$ and $\mathrm{I}_{A}$ ), local dendritic spikelets, and global dendritic spikes. For a comprehensive review of "dendritic democracy", rendering excitatory postsynaptic potential (EPSP) amplitudes at the soma insensitive to dendritic origin, we refer the reader to Häusser ([13]).

Our work is concerned with the analytical derivation of synaptic scaling rules that achieve dendritic democracy in purely passive models of branched dendrites. In particular, we ask: How must a synapse vary with distance to the soma in order that its impact on the somatic voltage be independent of its location? To make this question precise we need to model shunting synaptic currents. Postsynaptic shunting currents are induced by localized conductance changes associated with specific ionic membrane channels. The resulting currents are generally not proportional to the input conductance changes, and the conversion from conductance changes to membrane potential response is a nonlinear process. We model this as the product of a conductance, with amplitude $g_{\sigma}$ and duration $T$, and a shunt, measuring the difference between the membrane voltage $v$ and the synaptic reversal potential $E[11,6,16,31]$.

There is common agreement that the efficacy of a synapse is determined by the amplitude and duration of the resulting somatic depolarization (see [2] for a detailed discussion). We quantify the impact of synaptic input via the 5 


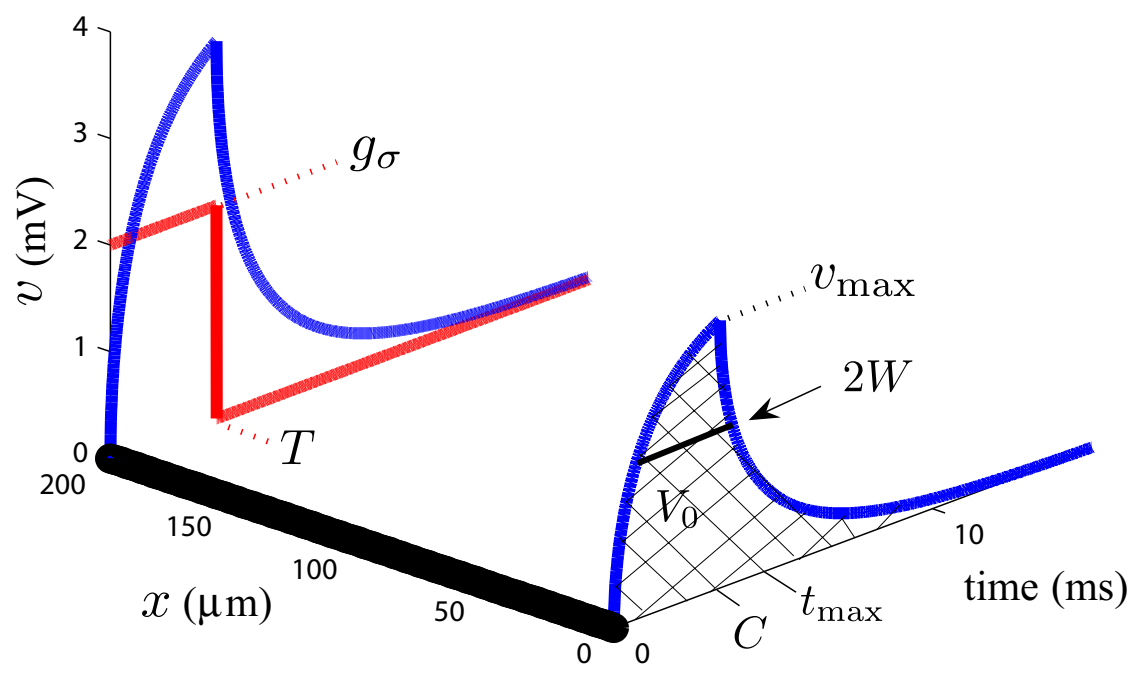

Figure 1: A segment of the neuron (heavy line) depicting the synaptic conductance (square wave of amplitude $g_{\sigma}$ and duration $T$ ), and synaptic $(x=200 \mu \mathrm{m})$ and somatic $(x=0)$ potentials. The latter is shorter and broader than the former on account of the cable filter. We quantify the somatic potential in terms of the strength, or area, $V_{0}$, centroid, $C$, width, $W$, peak, $v_{\max }$, and time to peak, $t_{\max }$, of the cross-hatched region.

measures of somatic response illustrated in Fig. 1: 1) the strength, $V_{0}$, defined as the area under the graph of the somatic depolarization, 2) the centroid, $C$, of this area, 3) the width, $W$, of this area, 4) the peak depolarization, $v_{\max }$, and 5 ) the time, $t_{\max }$, at which this peak occurs. The first three of these measures are moment based and discussed more fully in [2, 25], while the peak amplitude is used, for instance, in [19]. The peak, $v_{\max }$, captures the amplitude, $C, W$ and $t_{\max }$ speak to duration, and the strength, $V_{0}$, is a composite of amplitude and duration.

Presuming the soma to lie at $x=0$, and the synapse to be located at $x_{\sigma}$, the democratization problem now reduces to scaling $g_{\sigma}$ and $T$ with $x_{\sigma}$ in such a way that (combinations of) the aforementioned measures of the soma potential do not vary with $x_{\sigma}$. We find that for $x_{\sigma}$ close to the soma this scaling, with respect to $g_{\sigma}$, is linear, whilst further away it increases faster than linear. Interestingly, beyond a critical distance there is no choice of conductance strength that can lead to democracy.

Typically the mathematical treatment of shunting currents in dendritic 
systems is more involved than that of current injection, and is often abandoned in favor of a numerical analysis. Indeed previous work on synaptic scaling has relied upon compartmental modeling - see, for example, [18, 12, 22]. Here we discuss two mathematical approaches that circumvent the need for numerical simulations. The first technique equalizes the impact of spatially distributed synapses as measured by the moments of the somatic response (the first three measures of synaptic impact above). While this method is exact, its use in equalizing peak amplitude, that is measure 4), leads to a mathematically intractable problem. We therefore turn to a truncated Neumann series to construct the somatic response to shunting synaptic input. Even at second order we find excellent agreement with numerically obtained solutions of the full model within a physiologically realistic parameter set. We also contrast our findings with results achieved under the simpler hypothesis that synaptic activation results in current injections rather than conductance changes.

We start by a discussion of the cable models in $\S 2$. We describe the approach using moment based measures in $\S 3$ by constructing exact solutions to moments of the appropriate cable equation. Next, in $\S 4$, we show how to solve the model with shunts in terms of an infinite Neumann series. The first term of this expansion recovers the model without shunts (i.e. a simple current injection). A comparison of the series truncated at first and second order with numerically obtained solutions allows us to delimit their regimes of validity. Dendritic democracy which equalizes peak somatic response is discussed in $\S 5$. Finally, in $\S 6$, we discuss natural extensions of the work in this paper.

\section{Cable models and preliminary analysis}

The transmembrane potential, $v(x, t)$, relative to rest, at location $x$ and time $t$ along an infinite uniform passive cable with a synapse at $x=x_{\sigma}$, satisfies $[31,17]$

$$
\lambda^{2} v_{x x}(x, t)=\tau v_{t}(x, t)+v(x, t)+r g_{\sigma} H(T-t) \delta\left(x-x_{\sigma}\right)(v(x, t)-E),
$$

where the cell is initially at rest, i.e., $v(x, 0)=0$ for all $x$, and

$$
\lambda^{2}=\frac{a}{2 R_{i} G_{L}}, \quad \tau=\frac{C_{m}}{G_{L}}, \quad r=\frac{1}{2 \pi a G_{L}},
$$


$a$ is the radius of the cable, $R_{i}$ is the specific cytoplasmic resistivity, $C_{m}$ is the membrane capacitance, and $G_{L}$ is the membrane conductance. Here $H$ is the Heaviside function, and $\delta$ denotes the Dirac-delta function, so that the synaptic current or shunting current is the product of a synaptic conductance, of amplitude $g_{\sigma}$ and duration $T$, and a driving force biased by the reversal potential (relative to rest), $E$.

If one ignores the influence of the local potential, $v$, on the synaptic current, then one arrives at

$$
\lambda^{2} u_{x x}(x, t)=\tau u_{t}(x, t)+u(x, t)-r g_{\sigma} H(T-t) \delta\left(x-x_{\sigma}\right) E,
$$

the model for direct current injection.

Clearly, the analysis of the autonomous Eq. (2) is significantly easier than that of the non-autonomous Eq. (1). Throughout this work we identify the parameter regime in which model (2) is a good approximation of (1), as well as when this approximation breaks down.

In order to solve Eq. (1), we note that, away from the synapse, $v$ satisfies

$$
\lambda^{2} v_{x x}(x, t)=\tau v_{t}(x, t)+v(x, t), \quad x \neq x_{\sigma},
$$

while at the synapse, $x=x_{\sigma}$, we have

$$
\lambda^{2}\left\{v_{x}\left(x_{\sigma}^{+}, t\right)-v_{x}\left(x_{\sigma}^{-}, t\right)\right\}=r g_{\sigma} H(T-t)\left(v\left(x_{\sigma}, t\right)-E\right) .
$$

In the absence of end effects we expect the two one-sided slopes, $v_{x}\left(x_{\sigma}^{ \pm}, t\right)$, to be equal and opposite, and, as we assume that $0<x<x_{\sigma}$, we write Eq. (4) as

$$
2 \lambda^{2} v_{x}\left(x_{\sigma}, t\right)=r g_{\sigma} H(T-t)\left(E-v\left(x_{\sigma}, t\right)\right) .
$$

For infinite $T$ we follow [31] and evaluate the Laplace transform of Eqs. (3)$(5)$

$$
\begin{aligned}
\lambda^{2} \hat{v}_{x x}(x, s) & =(s \tau+1) \hat{v}(x, s), \\
2 \lambda^{2} \hat{v}_{x}\left(x_{\sigma}, s\right) & =r g_{\sigma} E / s-r g_{\sigma} \hat{v}\left(x_{\sigma}, s\right) .
\end{aligned}
$$

It follows that,

$$
\hat{v}(x, s)=\frac{\gamma E}{s(\sqrt{s \tau+1}+\gamma)} \exp \left(\sqrt{s \tau+1}\left(x-x_{\sigma}\right) / \lambda\right)
$$


where $\gamma$ is the nondimensional synaptic conductance

$$
\gamma=\frac{r g_{\sigma}}{2 \lambda}=\frac{g_{\sigma} \sqrt{R_{i} / G_{L}}}{(2 a)^{3 / 2} \pi}
$$

We next invert (6) and arrive at the voltage at the synapse

$$
v\left(x_{\sigma}, t\right)=\frac{E \gamma^{2}\left\{1-\operatorname{erf}(\sqrt{t / \tau}) / \gamma-\exp \left(\left(\gamma^{2}-1\right) t / \tau\right) \operatorname{erfc}(\gamma \sqrt{t / \tau})\right\}}{\gamma^{2}-1}
$$

In the case that $T$ is finite we note that, as Eq. (1) has but one solution, the expression in (8) is the solution of Eq. (1) up to time T. Although we shall see in $\S 4$ that the solution for times greater than $T$ can be expressed in terms of a convolution of (8) and the free-space Green's function,

$$
G(x, t) \equiv \frac{\exp \left(-t / \tau-\tau x^{2} /\left(4 \lambda^{2} t\right)\right)}{\sqrt{4 \pi \lambda^{2} t / \tau}},
$$

it turns out that the exact moments of $t \mapsto v\left(x_{\sigma}, t\right)$ require only knowledge of (8) up to time $T$.

Finally we recall that the Green's function leads to an explicit expression for $u$, the solution of the direct current injection model, Eq. (2). In particular, with $t \wedge T \equiv \min \{t, T\},[30]$

$$
\begin{aligned}
u(x, t) & =\frac{r g_{\sigma}}{\tau} E \int_{0}^{t \wedge T} G\left(x-x_{\sigma}, t-p\right) \mathrm{d} p \\
& =\gamma E\left\{A\left(x-x_{\sigma}, t-t \wedge T\right)-A\left(x-x_{\sigma}, t\right)\right\},
\end{aligned}
$$

where

$$
\begin{aligned}
A(y, t) & =\frac{1}{2}\{\exp (-y / \lambda) \operatorname{erf}(y /(2 \lambda) \sqrt{\tau / t}-\sqrt{t / \tau}) \\
& -\exp (y / \lambda) \operatorname{erf}(y /(2 \lambda) \sqrt{\tau / t}+\sqrt{t / \tau})\} .
\end{aligned}
$$

\section{Moment Methods}

We recall that synaptic impact or efficacy upon the soma is typically captured by some measure of the amplitude and duration of the resulting somatic 
potential. We start by following [2] in choosing moment based measures. To begin, we denote the $n$-th moment in time of the potential $v(x, \cdot)$ at $x$ by

$$
V_{n}(x) \equiv \int_{0}^{\infty} t^{n} v(x, t) \mathrm{d} t .
$$

Recalling Fig. 1 we focus on three common combinations of the first three moments:

- the strength is simply the zeroth moment, $V_{0}(x)$,

- the characteristic time is the centroid, $C(x)=V_{1}(x) / V_{0}(x)$, and

- the dispersion, $D(x)=V_{2}(x) / V_{0}(x)-C^{2}(x)$, is the square of the width, $W$.

Beginning with the first, we construct an exact expression for the somatic strength, $V_{0}(0)$, in terms of the synaptic strength $V_{0}\left(x_{\sigma}\right)$. We then derive an exact expression for the latter in terms of the nondimensional synaptic amplitude, $\gamma($ see $(7))$ and nondimensional synaptic duration

$$
\eta \equiv T / \tau
$$

We then show, for fixed $\eta$, how to scale $\gamma$ with $x_{\sigma}$, up to a critical distance $x_{\sigma}^{\lim }$, so that the somatic strength remains at a specified value $\bar{V}_{0}$. We conduct similar analysis of both $C(x)$ and $D(x)$ and show how one may simultaneously democratize strength, via scaling of $\gamma$, and the characteristic time or dispersion via scaling of $\eta$.

\subsection{Democratization of Strength}

Our immediate goal is to determine the dependence of the somatic strength, $V_{0}(0)$, on the location, amplitude and duration of the synapse. The following discussion, summarized in Proposition 1, shows how to vary the amplitude $g_{\sigma}$ with the distance $x_{\sigma}$ of the synapse from the soma, so that the resulting impact, as measured by $V_{0}$, is independent of $x_{\sigma}$. We assume that membrane constants, as well as the duration of synaptic activation, as measured by $T$ and its nondimensional form $\eta$, do not vary.

Integrating both sides of (3) over time gives

$$
\lambda^{2} V_{0}^{\prime \prime}(x)=V_{0}(x)
$$


while integrating (5) reveals

$$
\lambda V_{0}^{\prime}\left(x_{\sigma}\right)=\gamma E T-\gamma \int_{0}^{T} v\left(x_{\sigma}, t\right) \mathrm{d} t .
$$

Since solutions of (14) must decay to zero away from $x_{\sigma}$ it follows that

$$
V_{0}(x)=V_{0}\left(x_{\sigma}\right) \exp \left(\left(x-x_{\sigma}\right) / \lambda\right),
$$

and so, recalling Eq. (13),

$$
V_{0}\left(x_{\sigma}\right)=\lambda V_{0}^{\prime}\left(x_{\sigma}\right)=\gamma E T-\gamma \int_{0}^{T} v\left(x_{\sigma}, t\right) \mathrm{d} t=E \tau Q(\gamma, \eta) .
$$

Here $Q$ is obtained by integrating (8) up to time $T$. More precisely,

$$
Q(\gamma, \eta)=\frac{\gamma^{2} q(\gamma, \eta)-\gamma \eta}{\gamma^{2}-1}
$$

where, with $m \equiv \gamma^{2}-1$,

$$
q(\gamma, \eta)=\int_{0}^{\eta}\{\operatorname{erf}(\sqrt{y})+\gamma \exp (m y) \operatorname{erfc}(\gamma \sqrt{y})\} \mathrm{d} y
$$

and

$$
\int_{0}^{\eta} \operatorname{erf}(\sqrt{y}) \mathrm{d} y=(\eta-1 / 2) \operatorname{erf}(\sqrt{\eta})+\exp (-\eta) \sqrt{\eta / \pi}
$$

and

$$
m \int_{0}^{\eta} \exp (m y) \operatorname{erfc}(\gamma \sqrt{y}) \mathrm{d} y=\exp (m \eta) \operatorname{erfc}(\gamma \sqrt{\eta})-1+\gamma \operatorname{erf}(\sqrt{\eta}) .
$$

This exact solution can now be used to choose $g_{\sigma}\left(x_{\sigma}\right)$ to render the somatic strength, $V_{0}(0)$, independent of synapse location, $x_{\sigma}$. From (15)-(16) we find that this will follow if $\gamma$ satisfies

$$
Q(\gamma, \eta)=\frac{\bar{V}_{0}}{E \tau} \exp \left(x_{\sigma} / \lambda\right)
$$

where $\bar{V}_{0}$ is the desired, fixed somatic strength. On observing that $Q(0, \eta)=$ 0 , that $\gamma \mapsto Q(\gamma, \eta)$ is increasing and defining

$$
Q^{*}(\eta) \equiv \lim _{\gamma \rightarrow \infty} Q(\gamma, \eta)=(\eta+1 / 2) \operatorname{erf}(\sqrt{\eta})+\exp (-\eta) \sqrt{\eta / \pi}
$$


we arrive at

Proposition 1. Assume that the reversal potential, $E$, and the space and time constants ( $\lambda$ and $\tau$ respectively) are fixed. If the desired somatic strength, $\bar{V}_{0}$, effective synaptic duration, $\eta=T / \tau$, and distance to the soma, $x_{\sigma}$, together obey

$$
\bar{V}_{0} \exp \left(x_{\sigma} / \lambda\right)<E \tau Q^{*}(\eta)
$$

then there exists a unique synaptic conductance, $g_{\sigma}\left(x_{\sigma}\right)$, such that the associated somatic strength is indeed $\bar{V}_{0}$.

In Fig. 2 we present this equistrength synaptic conductance, $g_{\sigma}\left(x_{\sigma}\right)$, as a function of location, for a model cell for which

$$
\begin{gathered}
\ell=1 \mathrm{~cm}, \quad R_{i}=100 \Omega \mathrm{cm}, \quad C_{m}=1 \mu \mathrm{F} / \mathrm{cm}^{2}, \\
G_{L}=0.3 \mathrm{mS} / \mathrm{cm}^{2}, \quad E=60 \mathrm{mV} .
\end{gathered}
$$

We present results for different, but fixed, fiber radii $a$, that span a natural range. We note in each case that $g_{\sigma}$ exhibits moderate linear growth (less than $1 \mathrm{nS} / 100 \mu \mathrm{m}$ ) when the synapse is within $500 \mu \mathrm{m}$ of the soma. Choosing from the middle of the range, e.g., $a=1 \mu \mathrm{m}$ and $g_{\sigma}=5 \mathrm{nS}$, corresponds to $\gamma \approx 0.19$.

We plot the associated synaptic and somatic potentials in Fig. 3 for $a=2 \mu \mathrm{m}$. We see that in order to achieve a strength of $10 \mathrm{mVms}$ at the soma the synaptic potentials peak at less than $6 \mathrm{mV}$, even at $450 \mu \mathrm{m}$. This democratization of somatic strength is achieved by a simultaneous decrease in somatic peak and increase in somatic width (see Fig. 3B).

Although (20) is exact, it does not yield a solution in closed form. Toward such an end we note that the right side of (20) is typically small, and so, for small $\gamma$, we may exploit

$$
Q(\gamma, \eta)=\eta \gamma-\beta \gamma^{2}+O\left(\gamma^{3}\right)
$$

where

$$
\beta=(\eta-1 / 2) \operatorname{erf}(\sqrt{\eta})+\exp (-\eta) \sqrt{\eta / \pi},
$$

If we now solve $\eta \widetilde{\gamma}-\beta \widetilde{\gamma}^{2}=\bar{V}_{0} \exp \left(x_{\sigma} / \lambda\right) /(E \tau)$ we find

$$
\widetilde{\gamma}\left(x_{\sigma}\right)=\frac{\eta-\sqrt{\eta^{2}-4 \beta \bar{V}_{0} \exp \left(x_{\sigma} / \lambda\right) /(E \tau)}}{2 \beta} \approx \frac{\bar{V}_{0}}{E T} \exp \left(x_{\sigma} / \lambda\right) .
$$



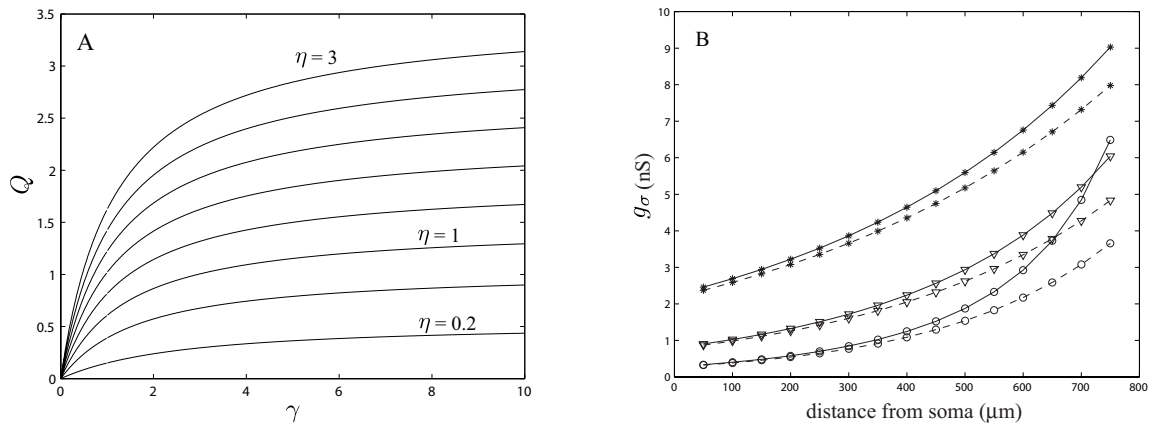

Figure 2: A: A plot of $Q(\gamma, \eta)$ as a function of the nondimensional synaptic conductance $\gamma$ (recall (16) and (17)) over modest $\gamma$. Results are presented for different values of the nondimensional synaptic duration $\eta$ ranging from 0.2 to 3 in steps of 0.4. B: The associated equistrength synaptic conductance, $x_{\sigma} \mapsto g_{\sigma}\left(x_{\sigma}\right)$, achieved by solving Eq. (20) for the cell described in (22)(23) with the specified somatic strength $\bar{V}_{0}=10 \mathrm{mVms}$ and $\eta=1$ and for several fiber radii: $a=2 \mu \mathrm{m}$ (asterisks), $1 \mu \mathrm{m}$ (triangles) and $0.5 \mu \mathrm{m}$ (circles). The associated dashed curves offer the approximations, final expression in Eq. (24), corresponding to the synaptic model, Eq. (2), of direct current injection.
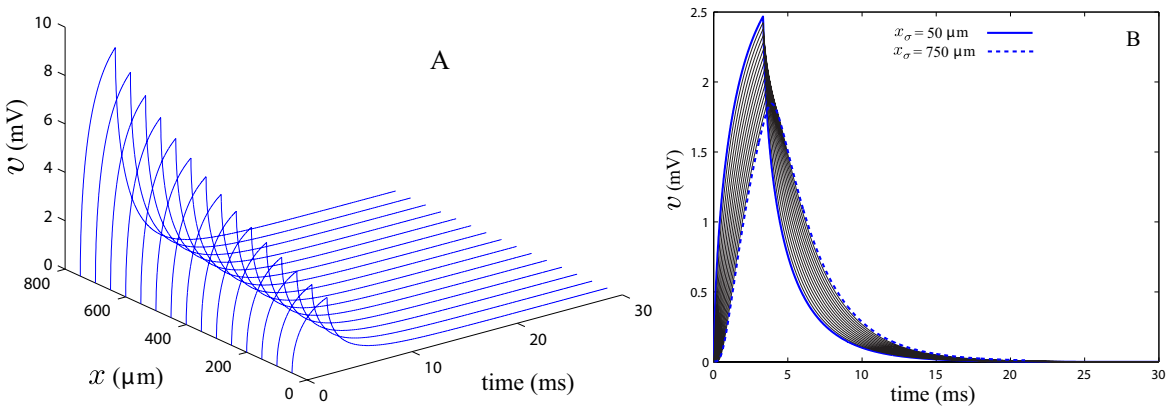

Figure 3: The computed synaptic (A) and somatic (B) potentials associated with the 'equistrength' synaptic conductance of Fig. $2 \mathrm{~B}$ in the case that $a=2 \mu \mathrm{m}, \bar{V}_{0}=10 \mathrm{mVms}$ and the remaining parameters given by (22)-(23). Regarding panel B we note that the somatic potentials become shorter and broader with increasing distance. 
We now show that this latter value is precisely the 'equistrength' synaptic conductance that holds in the simplified setting of Eq. (2) where the synaptic driving force is merely $E$. In this case the associated strength

$$
U_{0}(x) \equiv \int_{0}^{\infty} u(x, t) \mathrm{d} t=\gamma E T \exp \left(\left(x-x_{\sigma}\right) / \lambda\right)
$$

and so the associated equistrength conductance is $\bar{U}_{0} \exp \left(x_{\sigma} / \lambda\right) /(E T)$, as above. In Fig. 2 we contrast this conductance with the 'true' value obtained by numerically solving the implicit equation (20).

We next examine the limit of dendritic democratization of strength. The major difference between models (1) and (2) is that in the first case $v\left(x_{\sigma}, t\right)<$ $E$, regardless of $g_{\sigma}$, while in the second case the voltage, $u\left(x_{\sigma}, t\right)$, may be unbounded. In the limit $g_{\sigma} \rightarrow \infty$, the voltage $v\left(x_{\sigma}, t\right)$ is clamped to $E$ for $t \in[0, T]$ and decays exponentially afterwards. By monotonicity, the value of $V_{0}^{*}(0)$ corresponding to this profile cannot be exceeded by any other conductance change which is nonzero only in the interval $[0, T]$. Similarly, there is no value $g_{\sigma}$ that can result in a somatic strength $\bar{V}_{0}$ that exceeds this value $V_{0}^{*}(0)$.

Given a desired strength, we can use the results of this section to compute the location beyond which democratization fails. By Proposition 1, a prescribed somatic strength $\bar{V}_{0}$ can be achieved only for synapses located at a distance

$$
x_{\sigma}<\lambda \log \frac{E \tau Q^{*}(\eta)}{\bar{V}_{0}} \equiv x_{\sigma}^{\lim }
$$

from the soma. In Fig. 4 we show how this distance scales with radius, $a$, over a large range of values of intracellular resistivity, $R_{i}$ [12].

We emphasize that for model (2), associated with direct current injection, no such restriction on the achievable somatic strength exists. In this case, there is no mathematical limit to dendritic democracy. There are certainly physiological limits associated both with the number of postsynaptic receptors per synapse and, more importantly, the conductance beyond which the synaptic potential will activate sodium and/or potassium currents and so contradict the assumption of a passive cable.

\subsection{Democratization of Characteristic Time}

We have seen that democratization of strength via increasing synaptic amplitude, $g_{\sigma}$, alone leads to shorter but wider somatic responses. We now 


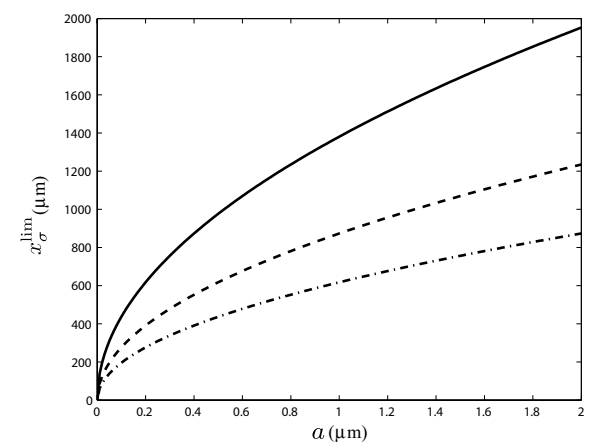

Figure 4: The point $x_{\sigma}^{\lim }$ of the limit of dendritic democracy as a function of the dendritic radius, $a$, for different values of $R_{i}: 100 \Omega \mathrm{cm}$ (solid), $250 \Omega \mathrm{cm}$ (dashed), and $500 \Omega \mathrm{cm}$ (dot dashed). All other parameters unchanged.

show how to scale the duration of the synaptic time course, $T$, in order to counteract this broadening. We accomplish this by focusing on two natural combinations of the first few moments of $t \mapsto v(x, t)$. With the synapse at $x_{\sigma}$ we first derive a simple law for transporting the synaptic characteristic time, $C\left(x_{\sigma}\right)$, to the somatic characteristic time, $C(0)$. We then show how to scale the duration of the synaptic input $T$ in order that $C(0)$ not vary with $x_{\sigma}$, assuming that $g_{\sigma}$ is fixed. We end the section by showing that $g_{\sigma}$ and $T$ can be scaled concurrently so that both $C(0)$ and $V_{0}(0)$ are independent of $x_{\sigma}$.

On taking the first moment of the two piece cable equation, (3) and (5), we find that $V_{1}(x)$ satisfies

$$
\lambda^{2} V_{1}^{\prime \prime}(x)=V_{1}(x)-\tau V_{0}(x) \quad \text { and } \quad \lambda V_{1}^{\prime}\left(x_{\sigma}\right)=\frac{\gamma E T^{2}}{2}-\gamma \int_{0}^{T} t v\left(x_{\sigma}, t\right) \mathrm{d} t .
$$

Therefore,

$$
V_{1}(x)=\left(V_{1}\left(x_{\sigma}\right)-\frac{V_{0}\left(x_{\sigma}\right) \tau\left(x-x_{\sigma}\right)}{2 \lambda}\right) \exp \left(\left(x-x_{\sigma}\right) / \lambda\right)
$$

and so

$$
\lambda V_{1}^{\prime}\left(x_{\sigma}\right)=V_{1}\left(x_{\sigma}\right)-\tau V_{0}\left(x_{\sigma}\right) / 2
$$

which, when reconciled with the above, yields,

$$
V_{1}\left(x_{\sigma}\right)=(\tau / 2) V_{0}\left(x_{\sigma}\right)+\frac{\gamma E T^{2}}{2}-\gamma \int_{0}^{T} t v\left(x_{\sigma}, t\right) \mathrm{d} t .
$$


It follows that the characteristic time obeys the very simple transport law

$$
C(x) \equiv \frac{V_{1}(x)}{V_{0}(x)}=C\left(x_{\sigma}\right)-\frac{\tau\left(x-x_{\sigma}\right)}{2 \lambda} .
$$

In order to democratize $C$ we ask that the somatic characteristic time, $C(0)$, take a prescribed value, call it $\bar{C}$, independent of $x_{\sigma}$. This compels us to solve $\bar{C}=C\left(x_{\sigma}\right)+\tau x_{\sigma} /(2 \lambda)$ and so we proceed to compute

$$
C\left(x_{\sigma}\right)=\tau R(\gamma, \eta)
$$

where

$$
R(\gamma, \eta)=\frac{1}{2}+\frac{\eta^{2} / 2-\gamma \int_{0}^{\eta} y\{\operatorname{erf}(\sqrt{y})+\gamma \exp (m y) \operatorname{erfc}(\gamma \sqrt{y})\} \mathrm{d} y}{\eta-\gamma \int_{0}^{\eta}\{\operatorname{erf}(\sqrt{y})+\gamma \exp (m y) \operatorname{erfc}(\gamma \sqrt{y})\} \mathrm{d} y} .
$$

Note that Eqs. (18) and (19) provide expressions for the integrals occurring in the denominator of $R(\gamma, \eta)$. Regarding the numerator, we record

$$
\int_{0}^{\eta} y \operatorname{erf}(\sqrt{y}) \mathrm{d} y=\operatorname{erf}(\sqrt{\eta})\left((2 \eta)^{2}-3\right) / 8+\exp (-\eta) \sqrt{\eta / \pi}(2 \eta+3) / 4
$$

and

$$
\begin{aligned}
& m^{2} \int_{0}^{\eta} y \exp (m y) \operatorname{erfc}(\gamma \sqrt{y}) \mathrm{d} y=1+\exp (m \eta)(m \eta-1) \operatorname{erfc}(\gamma \sqrt{\eta}) \\
& -\gamma m \exp (-\eta) \sqrt{\eta / \pi}+\gamma(m-2) \operatorname{erf}(\sqrt{\eta}) / 2 .
\end{aligned}
$$

It follows that $\eta \mapsto R(\gamma, \eta)$ is increasing and $R(\gamma, 0)=1 / 2$. As a consequence, we obtain a counterpart to Proposition 1

Proposition 2. If $\gamma$ is fixed and $1+x_{\sigma} / \lambda<2 \bar{C} / \tau$ then there exists a unique $T=T\left(x_{\sigma}, \bar{C}\right)$ such that the somatic characteristic time is $\bar{C}$.

We next turn to the simultaneous democratization of strength, $V_{0}(0)$ and characteristic time, $C(0)$. The discussion in the present and previous section shows that this is equivalent to solving the following equations simultaneously for $\gamma$ and $\eta$

$$
\begin{aligned}
Q(\gamma, \eta) & =\bar{V}_{0} \exp \left(x_{\sigma} / \lambda\right) /(E \tau) \\
R(\gamma, \eta) & =\bar{C} / \tau-x_{\sigma} /(2 \lambda) .
\end{aligned}
$$

We contrast the exact solution (via fsolve in Matlab) with an explicit approximate solution that stems from the observation that $\eta \mapsto R(\gamma, \eta)$ is very 
close to linear and very insensitive to $\gamma$. More precisely, $R(\gamma, \eta) \approx(1+\eta) / 2$ permits us to solve Eq. (29) for

$$
\eta\left(x_{\sigma} ; \bar{V}_{0}, \bar{C}\right) \approx 2 \bar{C} / \tau-\left(1+x_{\sigma} / \lambda\right)
$$

which we may then place in our approximate solution, Eq. (24), of Eq. (28), to yield

$$
\gamma\left(x_{\sigma} ; \bar{V}_{0}, \bar{C}\right) \approx \frac{\bar{V}_{0} \exp \left(x_{\sigma} / \lambda\right)}{E\left(2 \bar{C}-\tau\left(1+x_{\sigma} / \lambda\right)\right)} .
$$

Fig. 5 illustrates that these approximations faithfully follow the true solution.

We now tie this approximation back to the characteristic time of the potential, $u$, of the cable, Eq. (2), with direct current injection. First note that our approximation of $R$ when placed in Eq. (27) produces

$$
C\left(x_{\sigma}\right) \approx \frac{\tau+T}{2}
$$

Now, as in Eq. (26), we find that the first moment of $t \mapsto u(x, t)$ at $x_{\sigma}$

$$
U_{1}\left(x_{\sigma}\right)=\int_{0}^{\infty} t u\left(x_{\sigma}, t\right) \mathrm{d} t=\tau U_{0}\left(x_{\sigma}\right) /+\gamma E T^{2} / 2
$$

and so the associated synaptic characteristic time

$$
\frac{U_{1}\left(x_{\sigma}\right)}{U_{0}\left(x_{\sigma}\right)}=\frac{\tau+T}{2}
$$

indeed coincides with Eq. (32).

We have plotted in Fig. 6 the synaptic and somatic potentials associated with the true simultaneous democratizers, with $a=2 \mu \mathrm{m}$, and note that the addition of characteristic time has indeed reversed the trend noted in Fig. 3. That is, the peak somatic response now increases as a function of the distance of the synapse from the soma. Of course, this comes at the price of large, fast distal input and associated large synaptic potentials (see Fig. 6A). This characteristic time is not the only measure of somatic timing. In fact we shall find it useful to contrast the findings of this section with results of simultaneous democratization of strength and dispersion. 

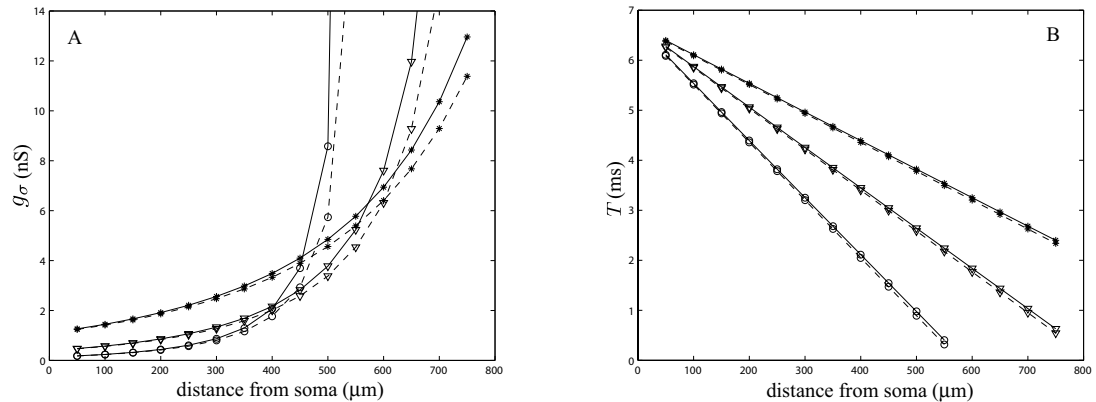

Figure 5: Simultaneous democratization of the fiber described in (22)-(23) with prescribed strength, $\bar{V}_{0}=10 \mathrm{mVms}$, characteristic time, $\bar{C}=3 \tau / 2$, and fiber radii: $a=2 \mu \mathrm{m}$ (asterisks), $1 \mu \mathrm{m}$ (triangles) and $0.5 \mu \mathrm{m}$ (circles). The solid lines in panels A and B correspond to the numerical solution of the full coupled system, (28) and (29), while the associated dashed curves offer the approximations, (30) and (31).
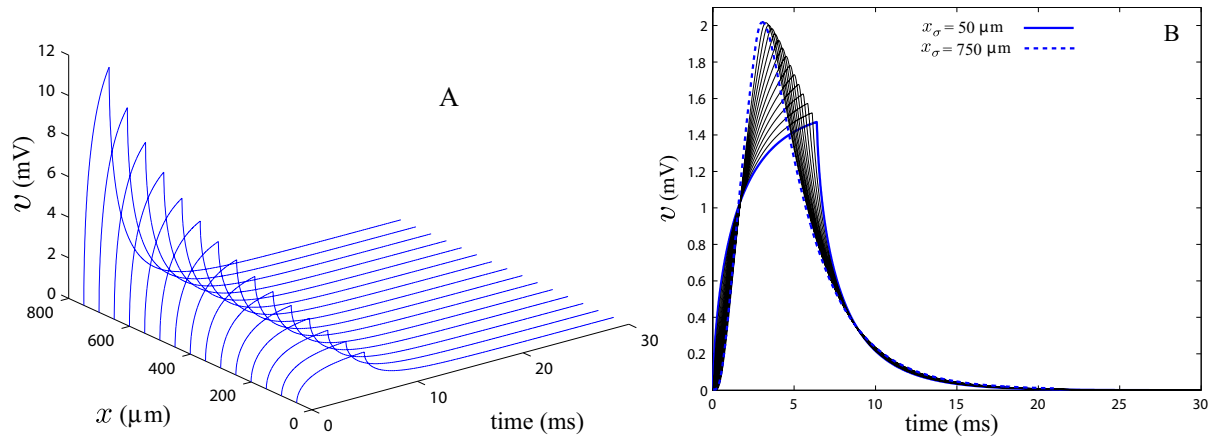

Figure 6: Synaptic (A) and somatic (B) potentials associated with simultaneous democratization of strength and characteristic time. All parameters are as in Fig. 5 with $a=2 \mu \mathrm{m}$. We note in panel B that the somatic potentials become taller and thinner with increasing distance. 


\subsection{Democratization of dispersion}

On computing the second moments of Eq. (3) and Eq. (5) we find that $V_{2}$ (defined in Eq. (12)) obeys

$$
\lambda^{2} V_{2}^{\prime \prime}(x)=V_{2}(x)-2 \tau V_{1}(x)
$$

subject to

$$
\lambda V_{2}^{\prime}\left(x_{\sigma}\right)=\gamma E T^{3} / 3-\gamma \int_{0}^{T} t^{2} v\left(x_{\sigma}, t\right) \mathrm{d} t
$$

It follows that

$$
\begin{aligned}
& V_{2}(x)=V_{2}\left(x_{\sigma}\right) \exp \left(\left(x-x_{\sigma}\right) / \lambda\right) \\
& -\left\{4 V_{1}\left(x_{\sigma}\right)+\tau\left(1-\left(x-x_{\sigma}\right) / \lambda\right) V_{0}\left(x_{\sigma}\right)\right\} \frac{\tau\left(x-x_{\sigma}\right)}{4 \lambda} \exp \left(\left(x-x_{\sigma}\right) / \lambda\right),
\end{aligned}
$$

where

$$
V_{2}\left(x_{\sigma}\right)=\tau^{2} V_{0}\left(x_{\sigma}\right) / 4+\tau V_{1}\left(x_{\sigma}\right)+\gamma E T^{3} / 3-\gamma \int_{0}^{T} t^{2} v(0, t) \mathrm{d} t .
$$

Therefore the dispersion

$$
D(x) \equiv \frac{V_{2}(x)}{V_{0}(x)}-\frac{V_{1}^{2}(x)}{V_{0}^{2}(x)}=D\left(x_{\sigma}\right)-\frac{\tau^{2}\left(x-x_{\sigma}\right)}{4 \lambda}
$$

obeys a very simple transport law. We proceed to compute

$$
D\left(x_{\sigma}\right)=\frac{V_{2}\left(x_{\sigma}\right)}{V_{0}\left(x_{\sigma}\right)}-C^{2}\left(x_{\sigma}\right)=\tau^{2} S(\gamma, \eta),
$$

where

$$
\begin{aligned}
& S(\gamma, \eta)=\frac{1}{4}+R(\gamma, \eta)-R^{2}(\gamma, \eta) \\
& +\frac{\eta^{3} / 3-\gamma \int_{0}^{\eta} y^{2}\{\operatorname{erf}(\sqrt{y})+\gamma \exp (m y) \operatorname{erfc}(\gamma \sqrt{y})\} \mathrm{d} y}{\eta-\gamma \int_{0}^{\eta}\{\operatorname{erf}(\sqrt{y})+\gamma \exp (m y) \operatorname{erfc}(\gamma \sqrt{y})\} \mathrm{d} y} .
\end{aligned}
$$

As is in the previous section, the denominator has been computed in Eqs. (18) and (19). Regarding the numerator, we record

$$
\begin{aligned}
& \int_{0}^{\eta} y^{2} \operatorname{erf}(\sqrt{y}) \mathrm{d} y=\operatorname{erf}(\sqrt{\eta})\left(\eta^{3} / 3-5 / 8\right) \\
& +\exp (-\eta) \sqrt{\eta / \pi}\left(\eta^{2}+5 \eta / 2+15 / 4\right) / 3
\end{aligned}
$$


and

$$
\begin{aligned}
& m^{3} \int_{0}^{\eta} y^{2} \exp (m y) \operatorname{erfc}(\gamma \sqrt{y}) \mathrm{d} y=\gamma \operatorname{erf}(\sqrt{\eta})\left(2-m+3 m^{2} / 4\right)-2 \\
& +\operatorname{erfc}(\gamma \sqrt{\eta}) \exp (m \eta)\left(m^{2} \eta^{2}-2 m \eta+2\right) \\
& +\gamma m(2-m(\eta+3 / 2)) \sqrt{\eta / \pi} \exp (-\eta) .
\end{aligned}
$$

We note that $S(\gamma, 0)=1 / 2$ and $\eta \mapsto S(\gamma, \eta)$ is increasing and so

Proposition 3. If $\gamma$ is fixed and $2+x_{\sigma} / \lambda<4 \bar{D} / \tau^{2}$ then there exists a unique $T=T\left(x_{\sigma}, \bar{D}\right)$ such that the somatic dispersion is $\bar{D}$.

As in the previous section, strength, $V_{0}(0)$ and dispersion, $D(0)$ can be democratized simultaneously by solving the pair of equations

$$
\begin{aligned}
Q(\gamma, \eta) & =\bar{V}_{0} \exp \left(x_{\sigma} / \lambda\right) /(E \tau) \\
S(\gamma, \eta) & =\bar{D} / \tau^{2}-x_{\sigma} /(4 \lambda) .
\end{aligned}
$$

We contrast the exact solution (via fsolve in Matlab) with an explicit approximate solution that stems from the observation that $\eta \mapsto S(\gamma, \eta)$ is very close to quadratic and very insensitive to $\gamma$. More precisely, $S(\gamma, \eta) \approx$ $1 / 2+\eta^{2} / 12$, which permits us to solve Eq. (36) for

$$
\eta\left(x_{\sigma} ; \bar{V}_{0}, \bar{D}\right) \approx \sqrt{12 \bar{D} / \tau^{2}-6-3 x_{\sigma} / \lambda},
$$

which we may then place in our approximate solution, Eq. (24), of Eq. (35), to yield

$$
\gamma\left(x_{\sigma} ; \bar{V}_{0}, \bar{D}\right) \approx \frac{\bar{V}_{0} \exp \left(x_{\sigma} / \lambda\right)}{E \tau \sqrt{12 \bar{D} / \tau^{2}-6-3 x_{\sigma} / \lambda}} .
$$

As above, these approximations faithfully follow the true solution (see Fig. 7). Moreover, the approximation jibes with the dispersion of the potential, $u$, of the cable, Eq. (2), with direct current injection. First note that our approximation of $S$ when placed in Eq. (34) produces

$$
D\left(x_{\sigma}\right) \approx \frac{6 \tau^{2}+T^{2}}{12} .
$$

Now, as in Eq. (33), we find that the second moment of $t \mapsto u(x, t)$ at $x_{\sigma}$ satisfies

$$
U_{2}\left(x_{\sigma}\right)=\int_{0}^{\infty} t^{2} u\left(x_{\sigma}, t\right) \mathrm{d} t=\gamma E T^{3} / 3+\tau U_{1}\left(x_{\sigma}\right)+\tau^{2} U_{0}\left(x_{\sigma}\right)
$$



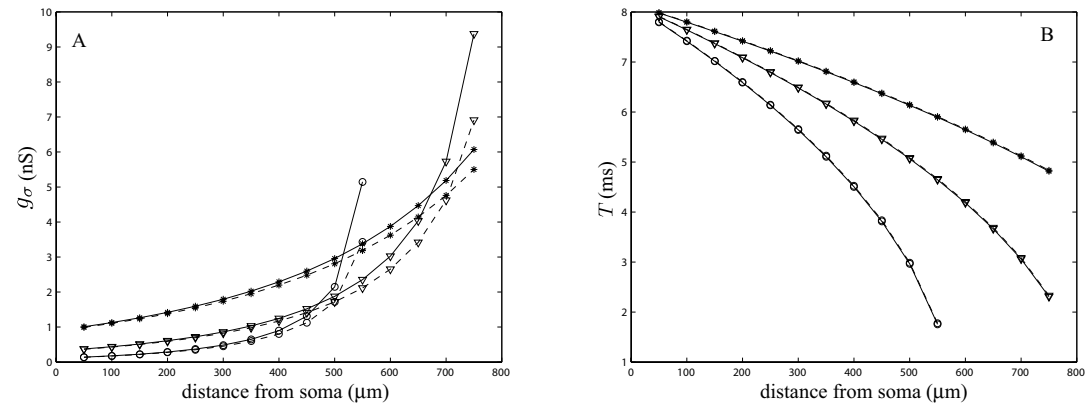

Figure 7: Simultaneous democratization of the fiber described in (22)-(23) with prescribed strength, $\bar{V}_{0}=10 \mathrm{mVms}$, dispersion, $\bar{D}=\tau^{2}$, and fiber radii: $a=2 \mu \mathrm{m}$ (asterisks), $1 \mu \mathrm{m}$ (triangles) and $0.5 \mu \mathrm{m}$ (circles). The solid lines in panels $\mathrm{A}$ and $\mathrm{B}$ correspond to the numerical solution of the full coupled system, (35) and (36), while the associated dashed curves offer the approximations, (37) and (38). We note that the solid and dashed curves coincide in panel $\mathrm{B}$.

and so the associated synaptic dispersion

$$
\frac{U_{2}\left(x_{\sigma}\right)}{U_{0}\left(x_{\sigma}\right)}+\frac{U_{1}^{2}\left(x_{\sigma}\right)}{U_{0}^{2}\left(x_{\sigma}\right)}=\frac{6 \tau^{2}+T^{2}}{12}
$$

indeed coincides with Eq. (39).

We have plotted in Fig. 8 the synaptic and somatic potentials associated with the true simultaneous democratizers, with $a=2 \mu \mathrm{m}$, of strength and diffusion. In comparison with the associated plots for the democratization of strength and characteristic time we note that the synaptic peaks and the variation in somatic peak with distance are both diminished. In addition, on comparing Fig. 5 to Fig. 7, we see that democratization of dispersion requires less drastic growth in $g_{\sigma}$ and decay in $T$ with distance.

Despite the above, in all instances of "democracy" we see that $g_{\sigma}$ scales linearly with $x_{\sigma}$ for small $x_{\sigma}$. This is consistent with the scaling laws found from experiments [21]. With increasing $x_{\sigma}$ we find that the democratic choice of $g_{\sigma}$ increases faster than linear. Moreover, in all cases beyond some critical distance from the soma there is no choice of $g_{\sigma}$ that can achieve democracy. We could, of course, proceed to simultaneously democratize strength and sensible combinations, e.g., skew and kurtosis, of higher and higher moments in the hopes of getting finer and finer control on the variation in peak somatic 

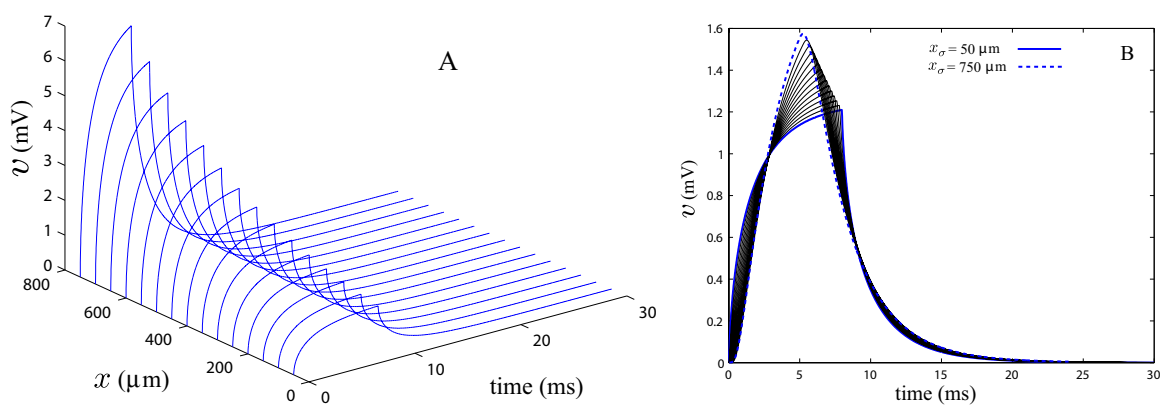

Figure 8: Synaptic (A) and somatic (B) potentials associated with simultaneous democratization of strength and dispersion. All parameters are as in Fig. 7 with $a=2 \mu \mathrm{m}$. We note in panel B that the somatic potentials become taller and thinner with increasing distance.

amplitude. However, we leave such an approach for future study, and instead embark on a more direct attack on the peak amplitude.

\section{Solution via a Neumann Series Expansion}

As we have seen in $\S 2$ cable theory shows that the potential induced by a synaptic current depends nonlinearly on the conductance change. As for the case of a constant current injection one can define a Green's function, though now it is no longer time-translation invariant, that can be used to find the cable voltage. However, for practical calculations it is more useful to formulate the cable response in terms of a Dyson equation. In this way we can express the response to synaptic current in terms of the bare response function of the model without shunting. For a further discussion of this useful relationship we refer the reader to the review by Bressloff and Coombes ([5]).

We begin by recasting the cable equation, Eq. (1), as an integral equation

$$
v(x, t)=\frac{r g_{\sigma}}{\tau} \int_{0}^{t \wedge T} G\left(x-x_{\sigma}, t-p\right)\left(E-v\left(x_{\sigma}, p\right)\right) \mathrm{d} p,
$$

where $G(x, t)$ is the Green's function of the infinite uniform passive cable expressed in Eq. (9). The implicit form of Eq. (41) suggests a Neumann series solution that can be obtained by repeated substitution of (41) into 
itself $[5,29]$. Performing a substitution just once, we have that

$$
\begin{aligned}
& v(x, t)=\frac{r g_{\sigma}}{\tau} E \int_{0}^{t \wedge T} G\left(x-x_{\sigma}, t-p\right) \mathrm{d} p \\
& -\left(\frac{r g_{\sigma}}{\tau}\right)^{2} E \int_{0}^{t \wedge T} G\left(x-x_{\sigma}, t-p\right) \int_{0}^{p} G\left(0, p-p^{\prime}\right) \mathrm{d} p^{\prime} \mathrm{d} p \\
& +\left(\frac{r g_{\sigma}}{\tau}\right)^{2} \int_{0}^{t \wedge T} G\left(x-x_{\sigma}, t-p\right) \int_{0}^{p} G\left(0, p-p^{\prime}\right) v\left(x_{\sigma}, p^{\prime}\right) \mathrm{d} p^{\prime} \mathrm{d} p .
\end{aligned}
$$

We recognize the first term as the exact solution, $u$, Eq. (10), to the problem of direct current injection. After an additional iteration we arrive at

$$
v(x, t)=u(x, t)-\frac{2 \lambda E \gamma^{2}}{\tau} \int_{0}^{t \wedge T} G\left(x-x_{\sigma}, t-p\right) \operatorname{erf}(\sqrt{p / \tau}) \mathrm{d} p+O\left(\gamma^{3}\right) .
$$

The integral term at second order can be obtained in closed form (via a Laplace transform) as

$$
\int_{0}^{t} G(y, t-p) \operatorname{erf}(\sqrt{p / \tau}) \mathrm{d} p=\tau B(y, t) /(2 \lambda)
$$

where

$$
\begin{aligned}
& B(y, t)=\frac{1}{2}\{\exp (-y / \lambda) \operatorname{erfc}(y /(2 \lambda) \sqrt{\tau / t}-\sqrt{t / \tau}) \\
& +\exp (y / \lambda) \operatorname{erfc}(y /(2 \lambda) \sqrt{\tau / t}+\sqrt{t / \tau})\} \\
& -\exp (-t / \tau) \operatorname{erfc}(y /(2 \lambda) \sqrt{\tau / t}) .
\end{aligned}
$$

Hence, the solution of Eq. (1) that includes only the first two terms in the Neumann series expansion is

$$
\begin{aligned}
& v_{N, 2}(x, t)=u(x, t)-E \gamma^{2} B\left(x-x_{\sigma}, t\right) \\
& +\frac{2 \lambda E \gamma^{2}}{\tau} \int_{0}^{\max \{0, t-T\}} G\left(x-x_{\sigma}, t-T-p\right) \operatorname{erf}(\sqrt{(p+T) / \tau}) \mathrm{d} p .
\end{aligned}
$$

We note that, although Tuckwell ([31]) has found an exact solution for $t<T$, the use of (43) is much preferred since it can be used to find the maximum of $v$ which always occurs at some $t \geq T$. It is useful to make a comparison between cable responses to synaptic input using numerical solutions to (1) 
(equivalent to a non-truncated Neumann series solution) and the approximate solutions $v_{N, 2}(x, t)$ and $u(x, t)$.

In Fig. 9 we plot the membrane voltage in the cable at the location of the synaptic input. The three curves in each plot correspond to the numerical solution of Eq. (1) (dashed black curve), the approximate solution $v_{N, 2}\left(x_{\sigma}, t\right)$ defined by Eq. (43) (red curve) and the solution $u\left(x_{\sigma}, t\right)$ given by Eq. (10) (blue curve). The numerical solution of the model was obtained using NEURON [7]. The model parameters are as in (22)-(23) with the cable radius, $a$ equal to $2 \mu \mathrm{m}$ in panel $\mathrm{A}, 1 \mu \mathrm{m}$ in panel $\mathrm{B}$, and $0.5 \mu \mathrm{m}$ in panel $\mathrm{C}$.

These plots nicely demonstrate that, for physiologically realistic parameter values, the approximate solution $v_{N, 2}\left(x_{\sigma}, t\right)$ is in very good agreement with the numerical solution of the model for relatively large values of the radius (Figs. 9A and $\mathrm{B}$ ). However, this is not the case when the radius of the cable is very small (Fig. 9C). Therefore for small radii more terms have to be included in the Neumann series expansion to improve accuracy. As expected, the solution $u\left(x_{\sigma}, t\right)$ of Eq. (2) offers a worse approximation than $v_{N, 2}\left(x_{\sigma}, t\right)$.

\subsection{Current injection versus conductance change}

As shown above, the solution of the model with shunting synaptic input (1) can be written as a Neumann series in the nondimensional synaptic conductance $\gamma$. If $\gamma \ll 1$, the contributions of the second and higher order terms in the expansion become negligible and the approximate solution is given by just the first term in (42). In this case the model with shunts will behave like the model without shunts given by Eq. (2). For larger values of the parameter $\gamma$ higher order terms in the Neumann series expansion cannot be ignored. As $\gamma$ increases the second order correction becomes more important, up to the point where $\gamma$ is so large that the Neumann series approximation $v_{N, 2}(x, t)$ breaks down.

For fixed values of $R_{i}$ and $G_{L}, \gamma$ is proportional to the ratio $g_{\sigma} /(2 a)^{3 / 2}$. Clearly, this dependence of $\gamma$ on the radius of the cable explains the results of Fig. 9 where both the full and approximate solutions are plotted for different values of $a$ (showing worse agreement with decreasing $a$ ). To explore the parameter range in which the Neumann series approximations $v_{N, i}$ are accurate we calculate the $L^{2}$ norms of their difference from the full solution of Eq. (1). In particular, we plot $\left|v\left(x_{\sigma}, t\right)-v_{N, i}\left(x_{\sigma}, t\right)\right|_{L^{2}}=$ $\left(\int_{0}^{\infty}\left[\left(v\left(x_{\sigma}, t\right)-v_{N, i}\left(x_{\sigma}, t\right)\right]^{2} \mathrm{~d} t\right)^{1 / 2}\right.$ for $i=1$ and 2 in Fig. 10 as functions of 

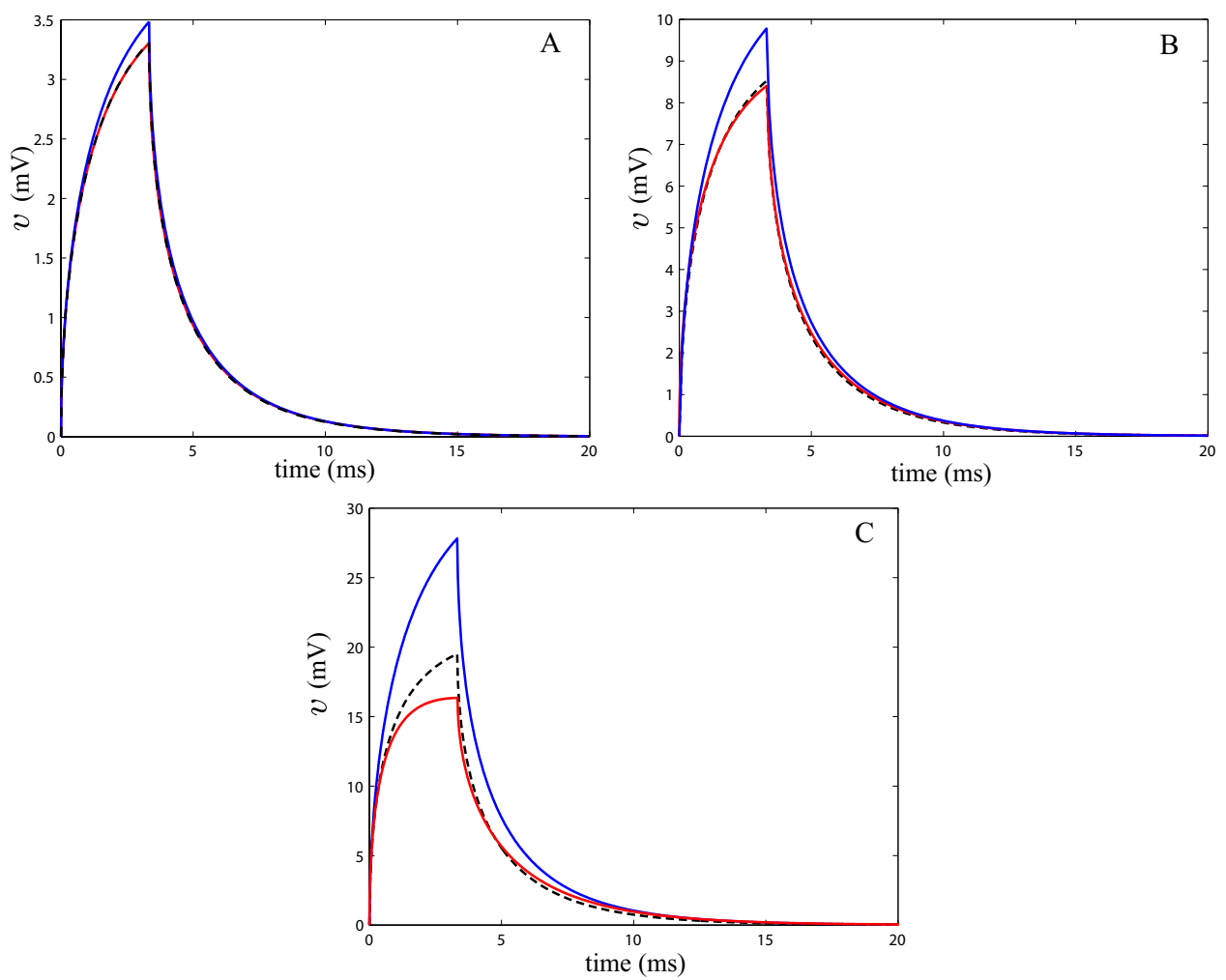

Figure 9: Comparison between the numerical solution of Eq. (1) (dashed black curve), the approximate solution $v_{N, 2}\left(x_{\sigma}, t\right)$ (red curve) and the exact solution $u\left(x_{\sigma}, t\right)$ of Eq. (2) (blue curve) for different values of the cable radius. The radius varies as A: $a=2 \mu \mathrm{m}, \mathrm{B}: a=1 \mu \mathrm{m}$ and $\mathrm{C}: a=0.5 \mu \mathrm{m}$. The synaptic duration $T=\tau$ and the synaptic conductance $g_{\sigma}=3 \mathrm{nS}$. Other parameters are as given in (22)-(23). 
$\gamma$ with fixed $g_{\sigma}$. The blue and red curves correspond to the case $i=1$ and $i=2$, and $v_{N, 1}\left(x_{\sigma}, t\right)=u\left(x_{\sigma}, t\right)$ and $v_{N, 2}\left(x_{\sigma}, t\right)$ respectively. The inner plot in this figure is a magnified view of the area marked by a dashed rectangle, and demonstrates that the error between the full (numerical) and approximate solutions can be significantly reduced (for relatively large radii) by adding the second term in the Neumann series expansion. However, if the radius of the cable is too small $\left(1 /(2 a)^{3 / 2}>2.5\right.$, i.e. $\left.a<0.272\right)$ solution $v_{N, 2}$ is actually worse than $v_{N, 1}$, and higher order terms in the Neumann series expansion are necessary.

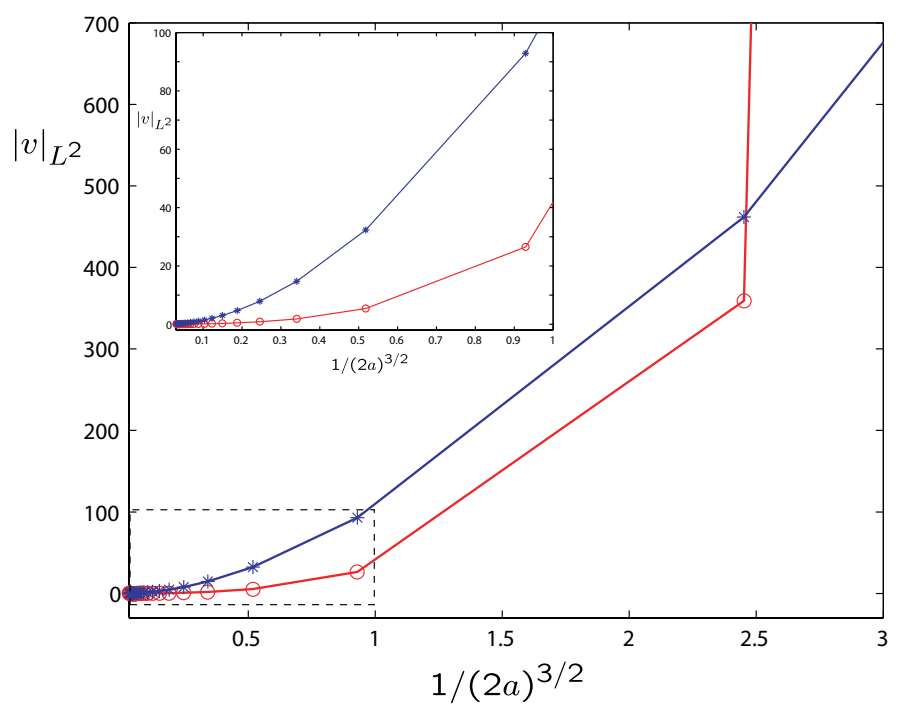

Figure 10: $L^{2}$ norms of the differences between the full (numerical) solution of Eq. (1) and the approximate solutions $v_{N}$ as a function of $\gamma$ with fixed $g_{\sigma}$. Solutions are compared at the location of the synaptic input $x_{\sigma}$. Blue curve: the approximate solution $v_{N}=u\left(x_{\sigma}, t\right)$. Red curve: the approximate solution $v_{N}=v_{N, 2}\left(x_{\sigma}, t\right)$. Parameters as in Fig. 9. Inner plot is a magnified view of the area marked by a dashed rectangle.

In Fig. 11 we use the data of Fig. 10 (for fixed $g_{\sigma}$ ) to quantify the parameter region in which the second order expansion is accurate (by demanding the solution satisfy $\left.|v|_{L^{2}}<0.85\right)$. For example with $\left(g_{\sigma}, a\right)=(5,2.5)$ we see that using just one term in the Neumann series expansion will give a poor approximation of the full solution, but that going to second order will give a good approximation. 

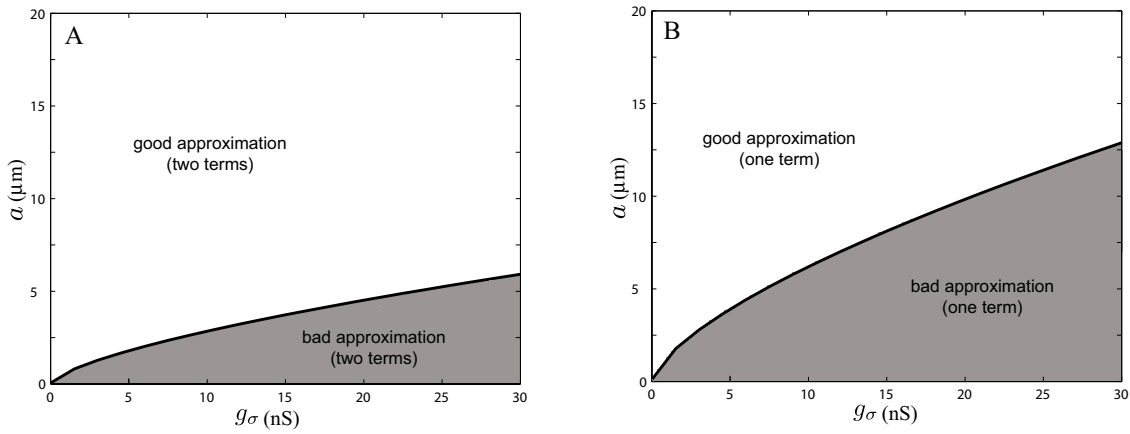

Figure 11: Regions of the parameter plane $\left(g_{\sigma}, a\right)$ in which the $L^{2}$ norm of the difference between the full solution and the approximate solution, $|v|_{L^{2}}$, is bigger (less) than 0.85, describing a bad (good) approximation. The gray area in A (B) corresponds to $v_{N, 2}(u)$ providing a bad approximation. Hence, using the Neumann series expansion, the region in the $\left(g_{\sigma}, a\right)$ plane supporting a good approximation to the full solution increases with the inclusion of higher order terms, as expected.

\section{Democracy: equalizing somatic response}

In this section we use the second order Neumann series approximation to determine the scaling of the synaptic strength $g_{\sigma}$ and duration $T$ necessary for "democratization." The impact of a synaptic input is characterized by the maximum of the depolarization at the soma, unlike in $\S 3$ where moment based measures were used. We first find conditions on $g_{\sigma}$ such that the maximum depolarization is independent of changes in the location of the synaptic input. We next find conditions such that, in addition, the maximum also occurs at the same point in time, regardless of the location of the input.

\subsection{Equalizing voltage peak}

We first consider the problem of equalizing the maximal depolarization at the soma. The only parameter that is allowed to vary is $g_{\sigma}=g_{\sigma}\left(x_{\sigma}\right)$, the location-dependent synaptic strength. Our aim is to find a function $g_{\sigma}$ that will equalize the voltage peak at the soma located at $x=0$, i.e. we require $\max _{t>0}\left\{v_{N, 2}(0, t)\right\}=\bar{V}$ for any $x_{\sigma}$. The time $t^{*}$ at which the maximum voltage is reached satisfies $t^{*} \geq T$. This will depend on the location $x_{\sigma}$ and 
can be found by solving $\partial v_{N, 2}(0, t) / \partial t=0$, where $v_{N, 2}(0, t)$ is given by (43). The time $t^{*}$ for each location $x_{\sigma}$ satisfies $v_{N, 2}\left(0, t^{*}\right)=\bar{V}$. Thus, the scaling law for $g_{\sigma}=g_{\sigma}\left(x_{\sigma}\right)$ can be found by simultaneously solving the following system of equations

$$
\begin{aligned}
& v_{N, 2}\left(0, t^{*}\right)-\bar{V}=0 \\
& \left.\frac{\partial v_{N, 2}(0, t)}{\partial t}\right|_{t=t^{*}}=0
\end{aligned}
$$

for the pair $\left(g_{\sigma}, t^{*}\right)$. Figure 12A shows how the synaptic strength has to scale along the cable to equalize the maximum response at the soma. Since $T$ is fixed, the times $t^{*}$ at which these maximal responses occur increase with $x_{\sigma}$. An example of this is shown in Fig. 12B. In Fig. 13 we plot the synaptic
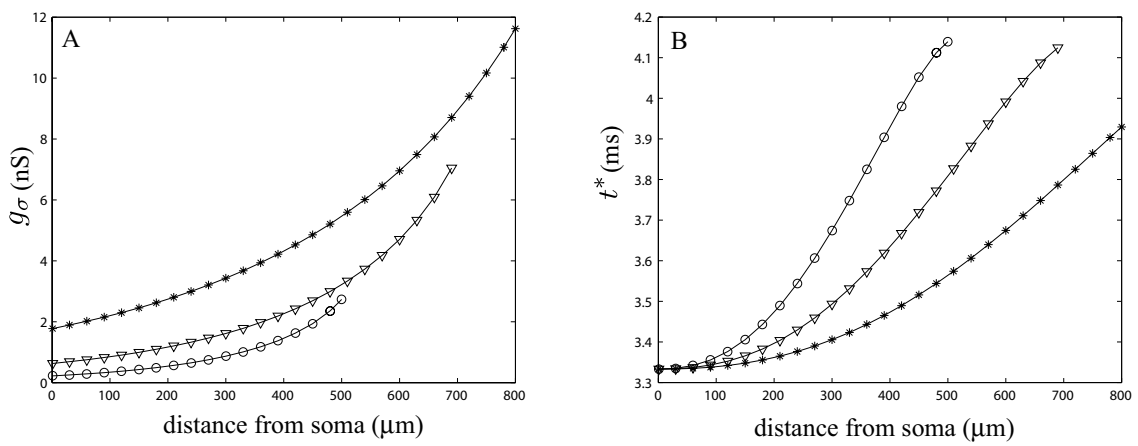

Figure 12: A: A plot of $g_{\sigma}=g_{\sigma}\left(x_{\sigma}\right)$ that "democratizes" the maximal response at the soma. B: A plot of $t^{*}=t^{*}\left(x_{\sigma}\right)$ (the time of maximal somatic response). Parameters as in Fig. 9 with $\bar{V}=2 \mathrm{mV}$ and $a=2 \mu \mathrm{m}$ (asterisks), $a=1 \mu \mathrm{m}$ (triangles), $a=0.5 \mu \mathrm{m}$ (circles).

and associated somatic potentials (Fig. 13A) and three examples of voltage profiles at the soma for different synaptic contact points (Fig. 13B). We note that the curves in Figs. 12 terminate at finite values of $x_{\sigma}$ because a solution to the pair of equations (44) and (45) ceases to exist. In parameter regimes where $v_{N, 2}$ is a poor approximation to the full solution this termination distance is an underestimate of the actual distance over which democracy can be guaranteed. 

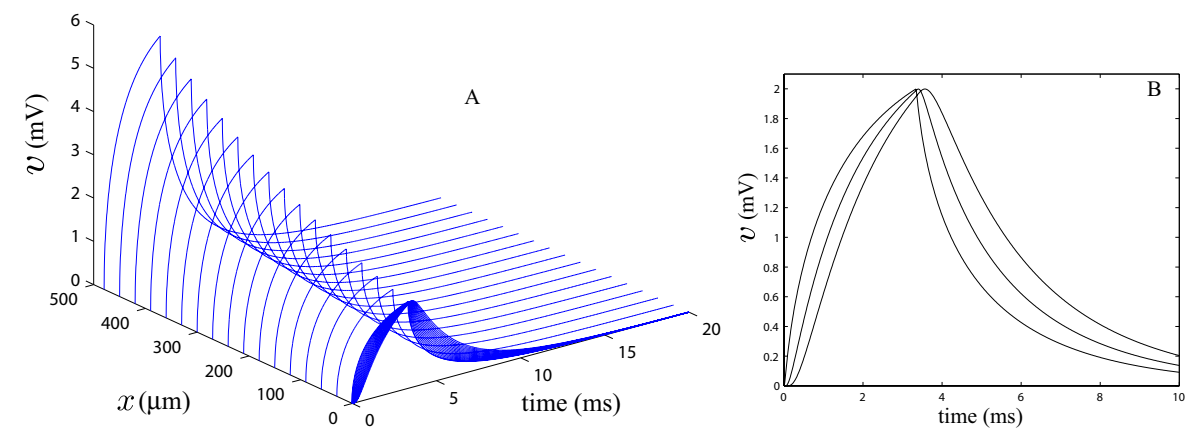

Figure 13: A: Synaptic and associated somatic potentials for a synaptic input at $x_{\sigma}$. B: Associated somatic potentials when the synaptic contact is at the distances of 90,290 and $490 \mu \mathrm{m}$ from the soma. Parameters as in Fig. 12 with $a=2 \mu \mathrm{m}$.

\subsection{Equalizing voltage peak and its time-to-peak}

We next examine the conditions under which the amplitude and time-to-peak at the soma is independent of the location of the synaptic input ( $t^{*}$ fixed). To accomplish this we let the duration of the synaptic input $T$ vary with the location of the input and write $T=T\left(x_{\sigma}\right)$. This more constrained form of "democracy" can be analyzed by solving (44) and (45) for $\left(g_{\sigma}, T\right)$. Results of such a calculation are shown in Fig. 14. In Fig. 15 we show the synaptic and associated somatic potentials (Fig. 15A) and three examples of voltage profiles at the soma for different synaptic contact points (Fig.15B).

As in $\S 3$, we see that "dendritic democracy" is ensured for $g_{\sigma}$ that scales linearly close to the soma and faster than linearly further away. This is seen in Figs. 12A and 14A, where it can also be seen that the critical distance beyond which democracy cannot be achieved decreases with decreasing cable radius. One may ask whether these results will hold for more complicated dendritic geometries relevant to real neurons. In the next section we introduce the appropriate mathematical techniques to answer this question and show that the answer is indeed yes.

\subsection{Branched dendritic structures}

Let us assume that we have an arbitrary branching dendritic structure and that the voltage dynamics $v_{i}(x, t)$ on each branch (with local spatial coor- 

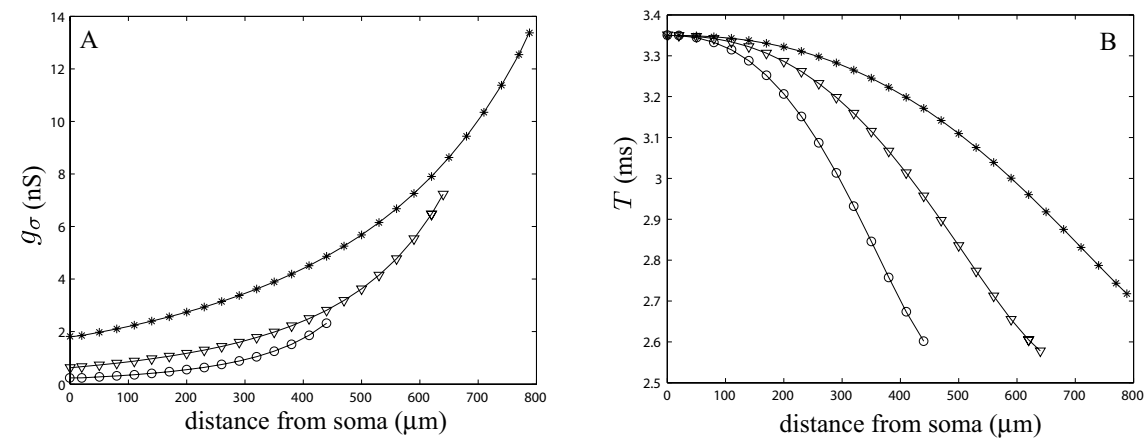

Figure 14: A plot of the functions $g_{\sigma}$ and $T$ (A and B) that equalize the maximum voltage and the time-to-peak at the soma. Here $t^{*}=3.35 \mathrm{~ms}$, $\bar{V}=2 \mathrm{mV}, a=2 \mu \mathrm{m}$ (asterisks), $a=1 \mu \mathrm{m}$ (triangles), $a=0.5 \mu \mathrm{m}$ (circles), and other parameters as in Fig. 9.
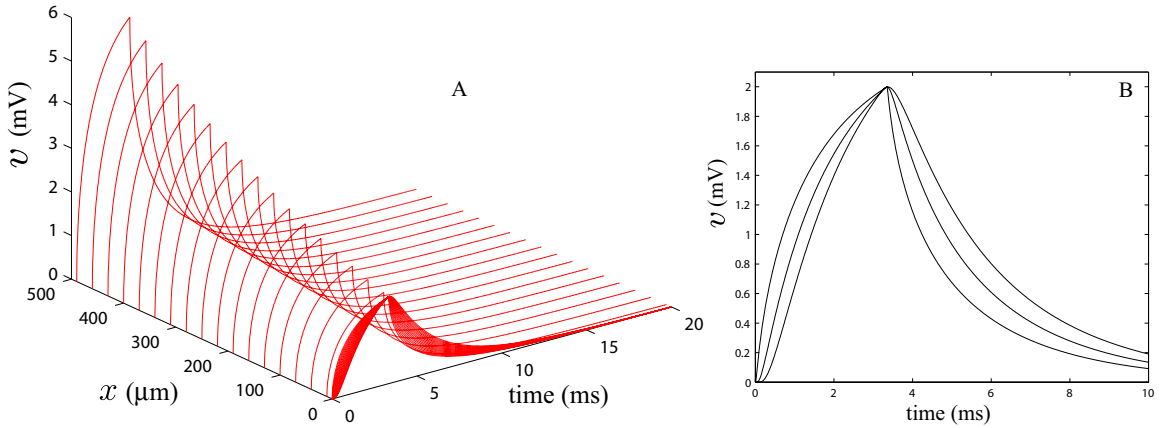

Figure 15: A: Synaptic and associated somatic potentials. B: Associated somatic potentials when synaptic contacts are at the distances of 90, 290 and $490 \mu \mathrm{m}$ from the soma. Parameters as in Fig. 14 with $a=2 \mu \mathrm{m}$. 
dinate $x$ ), labeled by $i$, is given by a passive cable equation. The synaptic conductance on branch $j$ at location $x_{j}$ is given as $g_{j}(t)=g_{\sigma} H(T-t)$. Then assuming $v_{i}(x, 0)=0$ for all branches, the voltage on each branch can be found as

$$
v_{i}(x, t)=\frac{r_{j}}{\tau_{j}} \int_{0}^{t} G_{i j}\left(x, x_{j}, t-s\right) g_{j}(s)\left(E-v_{j}\left(x_{j}, s\right)\right) \mathrm{d} s,
$$

where $G_{i j}\left(x, x_{j}, t\right)$ is the Green's function of the given branching structure. This can be constructed using the "sum-over-trips" framework [1], recently extended to tackle the inclusion of the soma and allowing for variation of parameters across branches [8]. Formally speaking if we denote the Green's function of an infinite cable by $G$ (Eq. (9)) then

$$
G_{i j}(x, y, t)=\sum_{\text {trips }} A_{\text {trip }} G\left(L_{\text {trip }}(i, j, x, y), t\right)
$$

where a "trip" depends on the labels $(i, j, x, y)$ and there are an infinite number of trips and trip coefficients $A_{\text {trip }}$. We refer the reader to $[1,8]$ for complete details. Following our earlier approach for an infinite cable we again pursue a Neumann series expansion. The first two terms in the series are

$$
\begin{aligned}
& v_{i}(x, t)=\left(\frac{r_{j} g_{\sigma}}{\tau_{j}}\right) E \int_{0}^{t \wedge T} G_{i j}\left(x, x_{j}, t-p\right) \mathrm{d} p \\
& -\left(\frac{r_{j} g_{\sigma}}{\tau_{j}}\right)^{2} E \int_{0}^{t \wedge T} G_{i j}\left(x, x_{j}, t-p\right) \int_{0}^{p} G_{j j}\left(x_{j}, x_{j}, p-p^{\prime}\right) \mathrm{d} p \mathrm{~d} p^{\prime} .
\end{aligned}
$$

The first term can be calculated using an infinite sum as $g_{\sigma} r_{j} E /\left(2 \lambda_{j}\right) \sum_{\text {trips }} A_{\text {trip }} K\left(L_{\text {trip }}(i, j, x, y), t\right)$, where $K(x, t)=A(x, t-t \wedge T)-$ $A(x, t)$ and $A(x, t)$ is given by (11). The second term is obtained from $G_{i j}(x, y, t)$ by numerical integration.

We now consider an example of a real neural geometry as shown in Fig. 16 and determine how the synaptic conductance scales along the apical trunk (shown in blue) to achieve (voltage) democracy. Further, we make a comparison between the full solution (obtained using NEURON) for the case where the synapse is modeled as in Eq. (1), the second order approximate solution given by (47), and the solution for the case where the synapse is modeled as in Eq. (2), so that the solution is simply the first term in (47). In Fig. 17 we show the democracy plots for the realistic neuron. It is apparent that the 


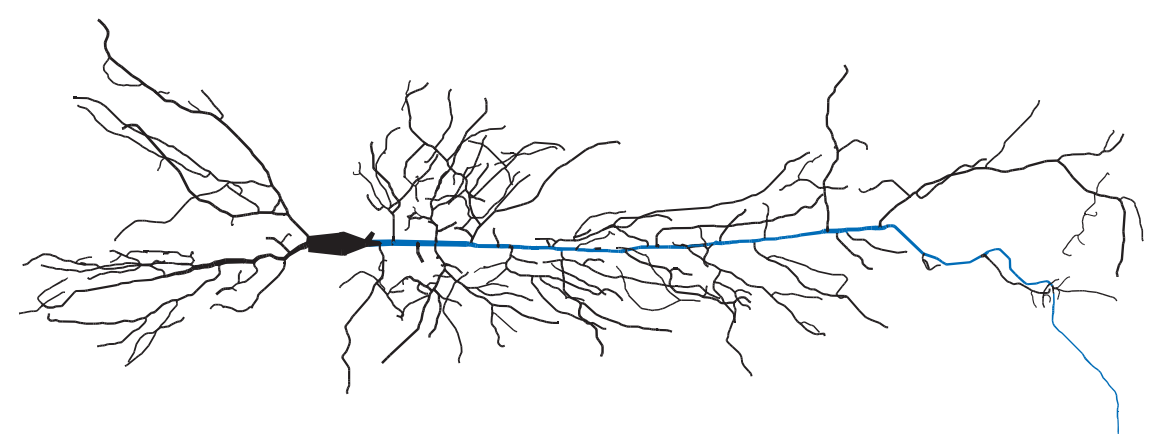

Figure 16: Reconstructed rat CA1 hippocampal pyramidal cell.
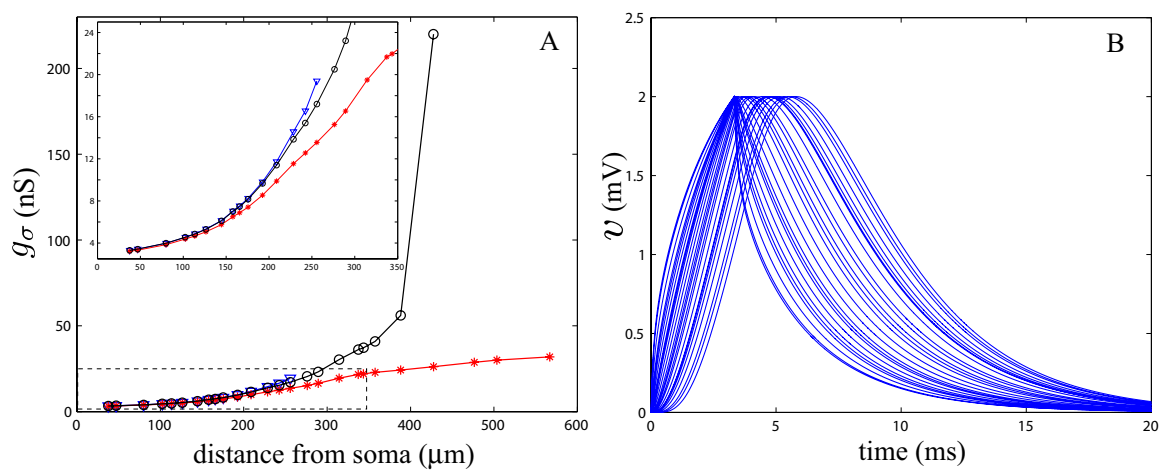

Figure 17: A: $g_{\sigma}$ as a function of distance (along the blue path in Fig. 16) that equalizes the maximum voltage $\bar{V}=2 \mathrm{mV}$ at the soma. Asterisks: first order approximation, circles: full numerical solution, triangles: second order approximation. Inner plot is a magnified view of the area marked by a dashed rectangle. Biophysical parameters across the tree are as given in (22)-(23) (with varying radii) and synaptic duration $T=\tau$. B: Voltage responses in the soma. 
first two terms of the Neumann series capture the behavior of the full model (obtained numerically), and that the trend in scaling is the same as for the unbranched case (cf. Fig. 12A).

The same trend can be observed for a more realistic shape of the synaptic conductance change, such as that of an alpha-function. Modeling the synaptic conductance on branch $j$ at location $x_{j}$ as $g_{j}(t)=g_{\sigma} t \exp (-\alpha t)$, the first term in a Neumann series expansion can be found as

$$
v_{i}(x, t)=-\frac{\mathrm{d}}{\mathrm{d} \alpha}\left(\frac{r_{j} g_{\sigma}}{\tau_{j}}\right) E \int_{0}^{t} G_{i j}\left(x, x_{j}, t-p\right) \exp (-\alpha p) \mathrm{d} p .
$$

The integral in (48) is easily calculated using the "sum-over-trips" prescription described above, under the replacement $1 / \tau \rightarrow 1 / \tau+\alpha$ in Eq. (11). Figure 18 compares the full solution (obtained numerically using NEURON) and the first order approximation given by (48).
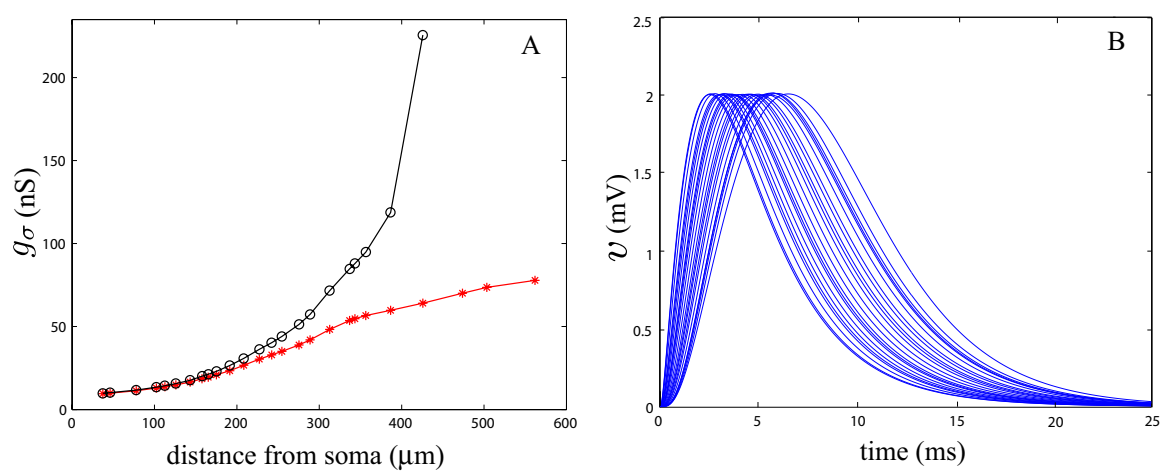

Figure 18: A: $g_{\sigma}$ as a function of distance (along the blue path in Fig. 16) that equalizes the maximum voltage $\bar{V}=2 \mathrm{mV}$ at the soma. Asterisks: first order approximation, circles: full numerical solution. Biophysical parameters across the tree are as given in (22)-(23) (with varying radii) and $\alpha=0.8$ $\mathrm{ms}^{-1}$. B: Voltage responses in the soma.

\section{Discussion}

In this paper we have focused on several key measures of "dendritic democracy" using two complementary approaches - one relying on moment methods and the other on a Neumann series solution. When combined, these two 
approaches suggest that the synaptic conductance has to scale linearly close to the soma and faster than linearly further away. Moreover, beyond some critical distance there is no choice of conductance that will ensure democracy in the presence of shunts. This suggests that, for distal synaptic inputs, democracy cannot be achieved by simply increasing synaptic conductance strength and that other mechanisms have to be invoked.

For example, the attenuation of voltage as it propagates from the distal dendrites to the soma might be compensated for by active currents. Indeed, dendritic P-type $\mathrm{Ca}^{2+}$ channels have already been studied as possible mediators of synaptic amplification via dendritic spikes to ensure democracy in a computational model of a cerebellar Purkinje cell [10]. Clearly, the inclusion of nonlinear membrane dynamics limits any quantitative mathematical analysis. However, recent work on the spike-diffuse-spike model has shown that this provides a reasonable caricature of a tree with active conductances and, importantly, is mathematically tractable [28, 29]. Our approach for studying "dendritic democracy" can be treated within this framework and will allow a systematic exploration of the amplifying nature of active spines on distal synaptic inputs. Moreover, it is believed that somatic response to distal inputs can be boosted not only by supra-threshold, but also by sub-threshold (resonant) dynamics, for example via $I_{h}$ and $I_{A}$ channels. The mathematical approach of this paper can be further extended to cover resonant branched dendrites using techniques developed in [8]. We note recent work showing that the tapering of real dendrites can equalize the current transfer from all synaptic locations [9]. It would be interesting to extend the present analysis to this case. On a final note, we emphasize that we have focused on single pulse stimulation, thus avoiding the complexities that arise when multiple stimuli interact. However, the work of Rall on spatio-temporal sequences of synaptic inputs [24] could easily be revisited within the framework we have presented here, and is a topic of current investigation.

\section{Acknowledgments}

The work in this paper is supported through EPSRC Grant No. GR/S60914/01. $\mathrm{S}$ Coombes would also like to acknowledge ongoing support from the EPSRC through the award of an Advanced Research Fellowship, Grant No. GR/R76219. K Josić was supported by NSF grant DMS-0604429, and an ARP/ATP grant. 


\section{References}

[1] L F Abbott, E Fahri, and S Gutmann, The path integral for dendritic trees, Biological Cybernetics 66 (1991), 49-60.

[2] H. Agmon-Snir, A novel theoretical approach to the analysis of dendritic transients, Biophysical Journal 69 (1995), no. 5, 1633-1656.

[3] P Andersen, H Silfvenius, S H Sundberg, and O Sveen, A comparison of distal and proximal dendritic synapses on CA1 pyramids in guinea-pig hippocampal slices in vitro, Journal of Physiology 307 (1980), 273-299.

[4] B K Andrasfalvy and J C Magee, Distance-dependent increase in AMPA receptor number in the dendrites of adult hippocampal ca1 pyramidal neurons, Journal of Neuroscience 21 (2001), 9151-9159.

[5] P.C. Bressloff and S. Coombes, Physics of the extended neuron, International Journal of Modern Physics B 11 (1997), 2343-2392.

[6] W. Burke, Spontaneous potentials in slow muscle fibres of the frog., J Physiol 135 (1957), no. 3, 511-21.

[7] N T Carnevale and M L Hines, The NEURON book, Cambridge University Press, 2006.

[8] S Coombes, Y Timofeeva, C-M Svensson, G J Lord, K Josić, S J Cox, and $\mathrm{C}$ M Colbert, Branching dendrites with resonant membrane: a "sum-over-trips" approach, Biological Cybernetics 97 (2007), 137-149.

[9] H Cuntz, A Borst, and I Segev, Optimization principles of dendritic structure, Theoretical Biology and Medical Modelling 4 (2007).

[10] E De Schutter and J M Bower, Simulated responses of cerebellar Purkinje cells are independent of the dendritic location of granule cell synaptic inputs, Proceedings of the National Academy of Sciences USA 91 (1994), 4736-4740.

[11] P. Fatt and B. Katz, An analysis of the end-plate potential recorded with an intracellular electrode., J Physiol 115 (1951), no. 3, 320-70. 
[12] N.L. Golding, T.J. Mickus, Y. Katz, W.L. Kath, and N. Spruston, Factors mediating powerful voltage attenuation along CA1 pyramidal neuron dendrites, The Journal of Physiology 568 (2005), no. 1, 69-82.

[13] Michael Häusser, Synaptic function: Dendritic democracy, Current Biology 11 (2001), R10-R12.

[14] R Iansek and S J Redman, The amplitude, time course and charge of unitary excitatory post-synaptic potentials evoked in spinal motoneurone dendrites, Journal of Physiology 234 (1973), 665-688.

[15] J J Jack, S J Redman, and K Wong, The components of synaptic potentials evoked in cat spinal motoneurones by impulses in single group Ia afferents, Journal of Physiology 321 (1981), 65-96.

[16] J.J.B. Jack, D. Noble, and R.W. Tsien, Electric Current Flow in Excitable Cells, Oxfor University Press, Oxford, UK, 1975.

[17] C. Koch, Biophysics of Computation: Information Processing in Single Neurons, Oxford University Press, 1999.

[18] M London and I Segev, Synaptic scaling in vitro and in vivo, Nature Neuroscience 4 (2001), 853-854.

[19] J C Magee, Dendritic integration of excitatory synaptic input, Nature Reviews Neuroscience 1 (2000), 181-190.

[20] J.C. Magee and E.P. Cook, Somatic EPSP amplitude is independent of synapse location in hippocampal pyramidal neurons, Nature Neuroscience 3 (2000), 895-903.

[21] M Migliore, M Ferrante, and G A Ascoli, Signal propagation in oblique dendrites of CA1 pyramidal cells, Journal of Neurophysiology 94 (2005), $4145-4155$.

[22] D.A. Nicholson, R. Trana, Y. Katz, W.L. Kath, N. Spruston, and Y. Geinisman, Distance-Dependent Differences in Synapse Number and AMPA Receptor Expression in Hippocampal CA1 Pyramidal Neurons, Neuron 50 (2006), no. 3, 431-442. 
[23] Daniel A Nicholson, Rachel Trana, Yael Katz, William L Kath, Nelson Spruston, and Yuri Geinisman, Distance-dependent differences in synapse number and AMPA receptor expression in hippocampal CA1 pyramidal neurons, Neuron 50 (2006), no. 3, 431-442.

[24] W Rall, Theoretical significance of dendritic trees for neuronal inputoutput relations, Neural Theory and Modeling, Stanford University Press, 1964.

[25] W Rall and H Agmon-Snir, Cable theory for dendritic neurons, Methods in Neuronal Modeling, 2nd ed., MIT Press, Cambridge, MA, 1998, pp. 27-92.

[26] C C Rumsey and L F Abbott, Equalization of synaptic efficacy by activity-and timing-dependent synaptic plasticity, Journal of Neurophysiology 91 (2004), 2273-2280.

[27] Clifton C Rumsey and L F Abbott, Synaptic democracy in active dendrites, Journal of Neurophysiology 96 (2006), no. 5, 2307-2318.

[28] Y Timofeeva, G J Lord, and S Coombes, Dendritic cable with active spines: a modeling study in the spike-diffuse spike framework, Neurocomputing 69 (2006), 1058-1061.

[29] Y Timofeeva, G J Lord, and S Coombes, Spatio-temporal filtering properties of a dendritic cable with active spines, Journal of Computational Neuroscience 21 (2006), 293-306.

[30] H C Tuckwell, Introduction to theoretical neurobiology: Linear cable theory and dendritic structure, Cambridge Studies In Mathematical Biology, vol. I, Cambridge University Press, 1988.

[31] H C Tuckwell, Introduction to theoretical neurobiology: Nonlinear and stochastic theories, Cambridge Studies In Mathematical Biology, vol. II, Cambridge University Press, 1988. 